Article

\title{
The Effects on Muscle Activity and Discomfort of Varying Load Carriage With and Without an Augmentation Exoskeleton
}

\author{
Huaixian $\mathrm{Li}^{1, *}$, Wenming Cheng ${ }^{1, *}$, Fang Liu ${ }^{1}$, Mingkui Zhang ${ }^{1}$ and Kun Wang ${ }^{2}$ \\ 1 School of Mechanical Engineering, Southewest Jiaotong University, \\ Chengdu 610031, Sichuan Province, China; liufang@swjtu.edu.cn (F.L.); sunson1688@163.com (M.Z.) \\ 2 School of Sports Medicine and Health, Chengdu Sport University, \\ Chengdu 610041, Sichuan Province, China; Michellebaobei@163.com \\ * Correspondence: ynlihuaixian@163.com (H.L.); wmcheng@home.swjtu.edu.cn (W.C.); \\ Tel.: +86-833-519-8015 (H.L.)
}

Received: 27 October 2018; Accepted: 11 December 2018; Published: 16 December 2018

\begin{abstract}
Load carriage is a key risk factor for Muscular Skeletal Disorders (MSDs). As one way to decrease such injuries, some exoskeletons have been developed for regular load carriage. We examined the ergonomic potential of an augmentation exoskeleton. Nine subjects completed eight trials of carrying tasks, using four loading levels $(0,15,30$, and $45 \mathrm{~kg})$ and two carrying conditions (with and without the exoskeleton). Electromyography (EMG) and the extended NASA-TLX rating scales were investigated and analyzed by linear mixed modeling and two-way ANOVA methods. Noraxon MR3.8, SPSS19.0, and MATLAB R2014b software were adapted. The results show that most of the muscle mean activities increased significantly $(p<0.05)$ with exoskeleton assistance. However, the interactive effects illustrate a decreasing trend with increase of load level. The mean discomfort rating scale values were generally higher, but subjects generally preferred using the exoskeleton in heavier loading tasks. The exoskeleton can effectively augment the performance of humans in heavy load carriage. The main reasons for higher muscle activity are from inflexible structures and inharmonious human-robot interactions. In order to decrease the MSD risks and increase comfort, optimal human-robot control strategies and adaptable kinematic design should be improved.
\end{abstract}

Keywords: exoskeleton; load carriage; muscle activities; human-robot interaction; discomfort

\section{Introduction}

Regular load carriages are criteria risk factors for Muscular Skeletal Disorders (MSDs) in hikers, backpackers, and soldiers [1,2]. The prevalence of MSDs is significantly related to the weight and mode of carrying a backpack among the load carrying population [3]. For military populations, high injury rates and high stress fracture rates have been associated with load carriage [1]. Knapik reported that while carrying heavy loads, 79 out of 335 infantry soldiers suffered from marching-related injuries in one road march [4].

Various preventive measures have been proposed for decreasing load carriage-related MSDs, such as mechanical aids like cranes [5]. However, with the development of new technologies, some potentially preventive strategies have emerged. One of these could be the use of wearable exoskeletons [6-8]. A wearable exoskeleton is defined as an active mechanical device, which is "worn" by an operator and fits closely to their body and works in concert with the operator's movements [9]. These newly developed exoskeletons proved to some extent helpful in decreasing the feeling of burden of the operators. Some positive study results reported the benefits of specific exoskeletons in reducing the internal muscle forces in corresponding body regions [8] and reducing high physical demands in 
standing status [10]. These exoskeletons have been designed to make load carriage easier by providing a parallel load path to the ground. These research results represent remarkable achievements in the field of load carrying magnitudes. However, the mean metabolic consumption and muscle force of human beings significantly increased despite the assistance of an exoskeleton [11]. This situation might be caused by kinematic misalignments between the complex human body structure and the simplified exoskeleton, since the augmentation devices were designed to adapt to the movement of human beings and to be "worn" by an operator. The design features determined that the augmentation devices could not self-stablilize and would start work in concert with the operator when they form a closed system [12]. Therefore, load carriage processes with an augmentation exoskeleton resulted in more load on human beings because of the adaptability to the movement of the human beings. The incompatibilities might actually increase the risks of muscular skeletal injuries, disorders, and discomforts as well. These potential problems are enormous challenges because they are influenced by some serious factors. For example, there are compromises to mobility, agility, and cognitive and physical performance in the human-exoskeleton interaction system with exoskeleton assistance, which may arise from aligning the exoskeleton to biological joints of human beings, the inertia compensation of the exoskeleton system [13], and adaptable control strategies of human-robot interactions. Therefore, in human-exoskeleton interaction processes, human beings have to spend energy and power to start, overcome hindrances, and stabilize the whole system. This might cause added load and fatigue.

Recently, a new series of studies reported a kind of elastic multi-joint soft exosuit which can help to decrease $7.3 \pm 5.0 \%$ of the metabolic power during carriage of a load equivalent to $30 \%$ human body mass. However, the lower limbs' electromyography (EMG) activity reduction in the experiment was not significantly different $(p<0.05)$. The small benefits have no clear effect on stress fractures and risk of sustaining injuries from heavy load carriage $[8,14]$. The soft exosuit needs no aligning to the human joints and overcomes most defects of the rigid exoskeletons. However, the device only augments the power of the operators' muscles and less is considered about the skeleton of the human beings and the injuries and MSDs which may happen in conditions of heavier loads and longer time of carriage.

Both of the above mentioned augmentation exoskeletons may form new kinds of risks of increasing Musculoskeletal Disorders and discomfort. To the authors' knowledge, however, there is still a lack of a readily available exoskeleton device for heavy load carriage to reduce muscular discomfort and the risk of MSDs. Most experiment studies were focused on oxygen consumption and muscle activity, with no further consideration to the discomfort, muscle activity patterns, and risk factors of MSDs [11].

Actual and virtual experiments in assessing the performance of the exoskeletons have already been undertaken $[15,16]$. In order to investigate the ergonomic performance of the augmentation exoskeleton developed by Southwest Jiaotong University, the effects on subjective discomfort and objective leg muscle activity are studied by a series of actual load carrying tasks. We investigated the subjective performance based on an extended questionnaire for discomfort and all six indices of the NASA TLX rating scale. We measured the surface electromyography signal of the knee extensor and ankle planter flexor to study the muscle activity patterns in the with-EXO condition. The results should display the ergonomic potentials of the augmentation exoskeleton. At the same time, the results might inspire kinematic design choices and optimal human-robot control strategies for exoskeletons in improving the usability and ergonomic comfort and decreasing the MSDs risk factors from the exoskeleton itself.

\section{Materials and Methods}

\subsection{Subjects and Prototypes}

In this study, nine healthy male participants (years: $25 \pm 8$; body mass: $71 \pm 12.4 \mathrm{~kg}$; height: $176 \pm 10 \mathrm{~cm}$ ) volunteered to take part in the study. None of the participants reported muscular-skeletal disorders in the previous three months. All subjects gave their informed consent for inclusion before 
they participated in the study. The study was conducted in accordance with the Declaration of Helsinki, and the protocol was reviewed and approved by the Ethics Committee of the Southwest Jiaotong University (approval number 2017_001).

A powered augmentation exoskeleton prototype, which was developed by the Southwest Jiaotong University, was used (as shown in Figure 1). The exoskeleton was described in detail by F.Liu and M.K.Zhang et al. $[17,18]$. The experimental prototype is the third generation prototype. Its mechanical structures consist of a backpack and two powered legs which power the knee joints via two hydraulic actuating cylinders. Using the servo valve to realize the servo-control for displacement of the hydraulic cylinder, the feedback Proportion-Integral-Derivative (PID) control law of the hydraulic cylinder displacement was designed to carry out force-assisted demands in a portable system under changing conditions of different weights and different poses. Each link could be adjusted to suit for the length of the human extremities. Additionally, the whole exoskeleton could be adjusted to suit human dimensions ranging from $165-185 \mathrm{~cm}$, covering $95 \%$ of the anthropometry population. Each leg has 6 degrees of freedom (DOFs): three hip joint DOFs, one knee joint DOF, and two ankle joint DOFs. The backpack is connected to the human trunk by shoulder girdles and a flexible waist bandage. The lower exoskeleton links are connected to the thighs by flexible bandages. Additionally, the operator's feet are put into the exoskeleton shoes.

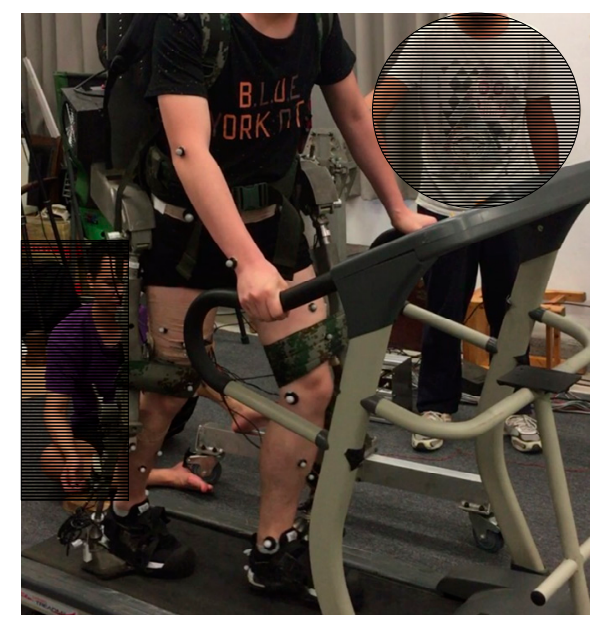

Figure 1. The argumentation exoskeleton prototype for load carriage experiments.

The exoskeleton is composed of rigid parts, two hydraulic cylinders, and some damping components, which are implanted in the exoskeleton to reduce vibration impacts. The Rigid-Flexible Coupling System has intrinsic compliance due to damping parts which allow for, and passively counteract, deviations from a target position in a safe way. Subjects were strapped to the exoskeleton by flexible, adaptable bandages, allowing free movement at the human joints [17].

The whole system control strategy was a kind of combination of position control and ZMP (zero moment point) control. The control scheme was shown in Figure 2. There are five different control strategies corresponding to five walking modes: level walking, level running, climbing slope, ladder walking, and standing. In order to study the basic muscle activities and subjective discomfort, we selected the level walking control mode to mimic free treadmill walking while carrying a load. 


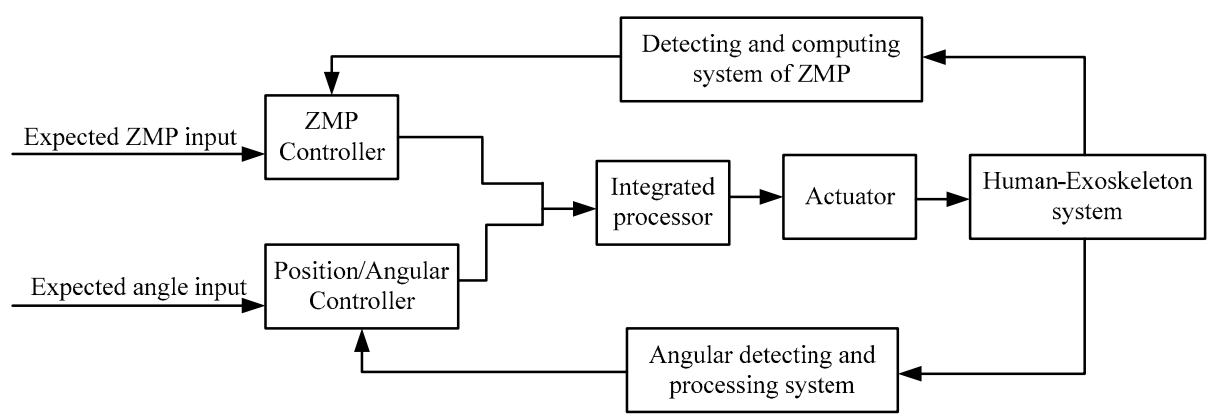

Figure 2. The overall control scheme.

\subsection{Testing Procedures}

Subjects completed two groups of contrast tasks, carrying $0 \mathrm{~kg}$, $15 \mathrm{~kg}, 30 \mathrm{~kg}$, and $45 \mathrm{~kg}$ weight in each group of trials, with and without the exoskeleton prototypes. All subjects had recently completed about 30 min basic training on the treadmill, walking with the exoskeleton and without loads in order to familiarize them with the device. Each participant performed 3 trials of 8 min walking in succession with 2 min rest between the trials. The training section had been carried out a week before the formal experiment. All volunteers were healthy and without muscular-skeletal injuries or disorders. We started with the group of trials without the exoskeleton, followed by the group of tasks with exoskeleton; the order of the two groups (with and without exoskeleton) within the tasks was assigned and based on a Latin square approach. The order of presentation of the load conditions was randomly assigned and based on a Latin square approach, as shown in Figure $3 \mathrm{~b}$. In order to keep the consistency of the trials, the subjects completed all of the trials with the help of the treadmill handlebars in case of falling down when carrying heavier loads. All sessions were performed in a laboratory at a constant ambient temperature of $22^{\circ} \mathrm{C}$.

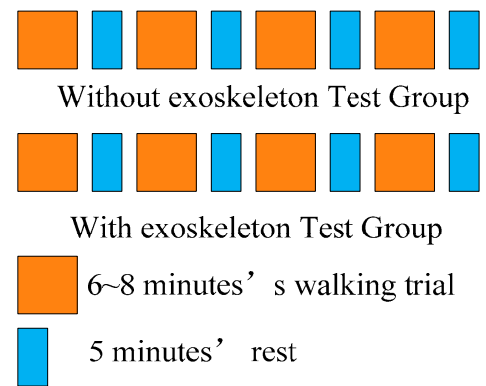

(a)

\author{
A B D C A- $0 \mathrm{~kg}$ loading \\ B C A D B- $15 \mathrm{~kg}$ loading \\ C D B A C- $30 \mathrm{~kg}$ loading \\ D A C B D- $45 \mathrm{~kg}$ loading
}

(b)

Figure 3. Process of the experiments. (a) Testing procedure: the orange square block means 6-8 min walking trial and the blue rectangle means 5 min rest; (b) the Latin square arrangement: the capital letters A, B, C, and D are used to represent the load level $0 \mathrm{~kg}, 15 \mathrm{~kg}, 30 \mathrm{~kg}$, and $45 \mathrm{~kg}$ separately.

Prior to testing, the exoskeleton was adjusted for each participant, ensuring that an acceptable fit was achieved, as determined independently by an exoskeleton engineer. The criteria was to keep the exoskeleton links fitting the length of the shank and thigh of each subject, as measured by a ruler and the engineer's experience. Each volunteer then completed a total of 6-8 min warm-up with the exoskeleton. According to the completed training session, the volunteers become familiar with carrying each load while wearing the exoskeleton whilst walking on the treadmill at $1 \mathrm{~m} / \mathrm{s}(3.6 \mathrm{~km} / \mathrm{h})$. Participants walked on the treadmill for $6 \mathrm{~min}$ in each trial at a speed of $3.6 \mathrm{~km} / \mathrm{h}$. At the beginning of the fourth minute of trial, $30 \mathrm{~s}$ data of human kinematics and muscle activity was collected; the signals used for controlling the exoskeleton were collected as well. It concerns about 12 walking cycles. After each trial was completed, all load components were removed and the volunteer was allowed approximately $5 \mathrm{~min}$ to rest between trials. 
After each test section, the participants were asked to rate a subjective questionnaire on a visual rating scale form. They marked their rating on seven linear rating scales that ranged from 0 (low) to 100 (high) points, as shown in Figure 4.

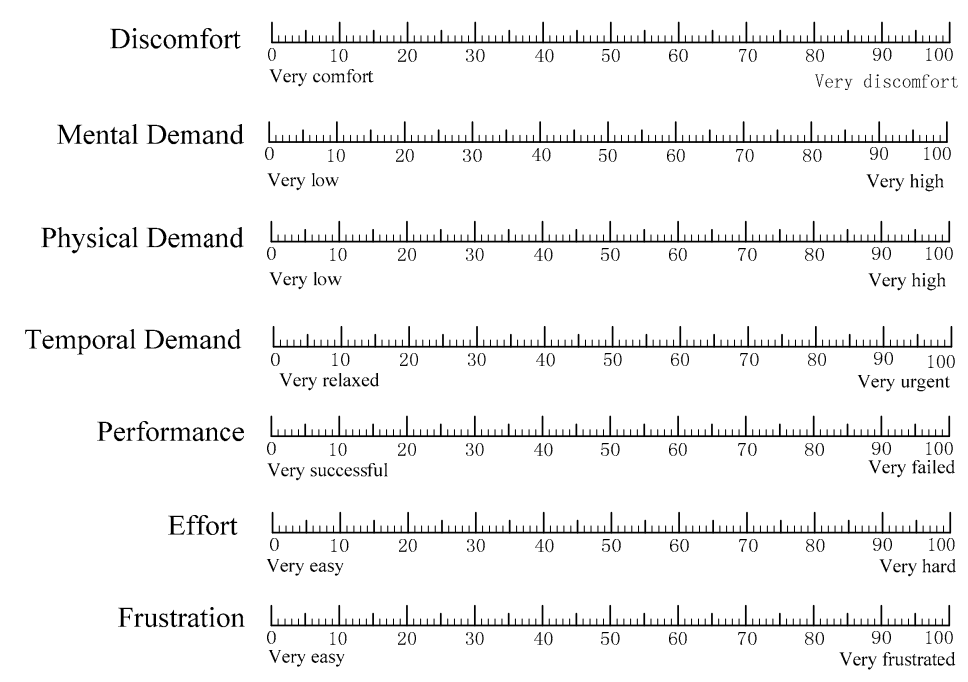

Figure 4. The extended NASA-TLX rating scales [19].

\subsection{Measurements}

The Noraxon Desktop DTS 8-channel wireless electromyography (EMG) system (Noraxon Ltd., Scottsdale, AZ, USA) was used in combination with bi-polar $\mathrm{Al} / \mathrm{AgCl}$ surface electrodes. EMG data were recorded through analysis software MR 3.8 (Noraxon Ltd., USA) at a sample rate of $2000 \mathrm{~Hz}$. EMG data of seven muscles on the right leg was recorded. The seven muscles are rectus femoris (RF), vastus medialis $(\mathrm{VM})$, vastus lateralis $(\mathrm{VL})$, semitendinosus $(\mathrm{ST})$, biceps femoris $(\mathrm{BF})$, gastrocnemius lateralis (GL), and peroneus longus (PL). Maximal voluntary contraction values (MVC) were also recorded. The MVC was realized by Isometric contraction. The participants sat in a self-contained chair designed by the Isomed2000 testing system. Knee joint angles of $105^{\circ}$ and $150^{\circ}$ were selected. Subjects performed a $6 \mathrm{~s}$ maximal voluntary contraction. The bi-polar surface electrodes were located in the positions shown in Figure 5, which complied with SENIAM recommendations (http:/ / www.seniam. org). Raw EMG signals were first filtered using a notch filter $(50 \mathrm{~Hz})$ to reduce effects of alternating current. Then a band-pass $(10-500 \mathrm{~Hz})$ was used to reduce potential effects of transitional adjustments. The integration was expressed as a percentage of the peak voltage of the MVCs. In each of these trials, EMG root mean square (RMS) values were obtained. Similar processing was done for raw EMG values obtained during reference contractions. Mean RMS values obtained during the task were then normalized (nRMS) to the corresponding reference RMS values.

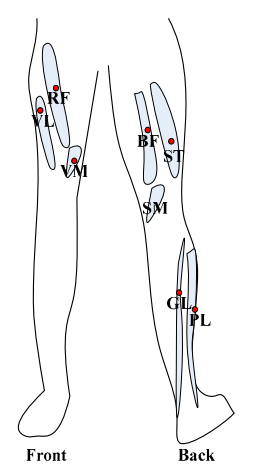

Figure 5. The body parts and points where the bi-polar surface electrodes were located. 
An extended NASA TLX rating scale is used to record the discomfort of the size indices [19]. After each subject complete the eight trials, the TLX rating scales were acquired.

\subsection{Subjective Performance and Discomfort}

The rating scale is an extended NASA TLX questionnaire with an extra scale of discomfort, as introduced by Hart and Stavenland. The discomfort index and the six standard indices were defined as the following [17]:

(1) Discomfort (C): How comfortable was the appointed loading during walking?

(2) Mental Demand (MD): How mentally demanding was the task?

(3) Physical Demand (PD): How physically demanding was the task?

(4) Temporal Demand (TD): How hurried was the pace of the task?

(5) Performance (OP): How successful were you in accomplishing the task?

(6) Effort (EF): How hard did you have to work to accomplish the task?

(7) Frustration (FR): How insecure, discouraged, irritated, stressed, and annoyed were you?

After all eight trials, the weighting factors for the standard rating scales were acquired for each subject by pair-wise comparison and computed into an overall set of group weights. At the same time, the subjects would be asked by the experimenter which body part experienced the most discomfort in the trials.

\subsection{Data Analysis}

Linear mixed modeling analyses were applied to explain differences in mean EMG activity over time by the factored type of walking ("with- or without-EXO") and load carriage (four-level factor "loading"). Differences in average EMG activation, subjective performance between the two walking types, and four loading tasks were therefore analyzed using a two-way ANOVA for repeated measures with independent factors for the exoskeleton (with or without) and loading (four-level factor "loading"). To evaluate the effects of the exoskeleton in the EMG gait patterns, unilateral gait pattern differences in two types of walking, four-level loading, and two-level factor intervals (stance and swing) were analyzed. A paired sample $t$-test was carried out for the loading tasks.

After carefully inspecting data, the Kolmogorov-Smirnov and Shapiro-Wilk test were performed. The NASA-TXL rating scores were not normally distributed. Differences between the two walking conditions (with or without exoskeleton) during the loading tasks were analyzed using Wilcoxon signed rank tests (i.e., using participants as their own controls). Significance was accepted at $p<0.05$ and all statistical analyses were performed using SPSS (IBM SPSS Statistics 19.0).

\section{Results}

The experimental results should be presented and briefly discussed in the following order: (1) Overall Stance and Swing durations, with- or without-Exo load carriage. (2) Overall gait pattern and characteristics, with- or without-EXO load carriage through a case study. (3) For the two independent variables, load and with- or without-Exo, the mean and maximum muscle activities from EMG data will be presented for the knee extensors (RF, VM, VL), knee flexors (ST, BF), and ankle planter flexors (PL, GL). (4) The two-way ANOVA interaction effects of load $\times$ with- or without-EXO are presented. (5) The NASA-TXL discomfort and standard subjective performance.

\subsection{The Overall Stance and Swing Duration for with- or without-Exo Carriage Condition}

As shown in Figure 6, the investigation of the stance and swing duration shows that the stance duration was prolonged with increased loading in the without-Exo condition. And in the with-Exo condition, the stance and swing durations were almost the same. However, the statistic results illustrated that there were no significant difference $(p>0.05)$ between with-Exo and without-Exo 
condition. The reason for the increase of the stance phase with the EXO might be the trigger time of the EXO since the exoskeleton must be started by human beings.

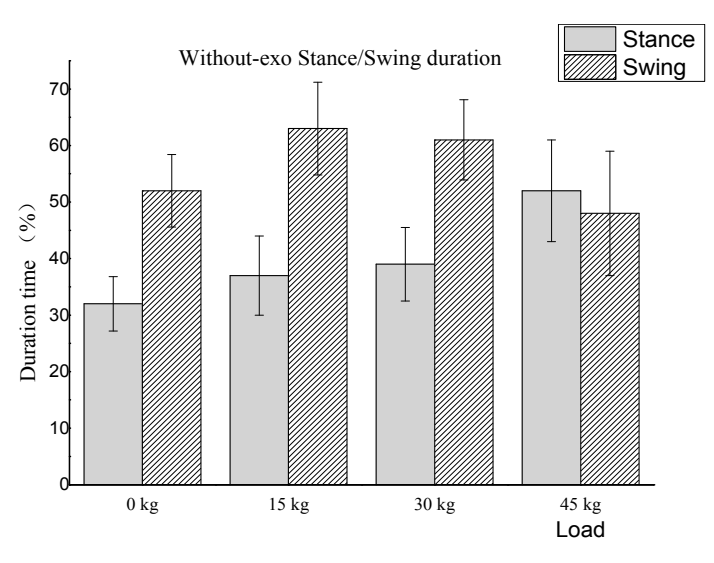

(a)

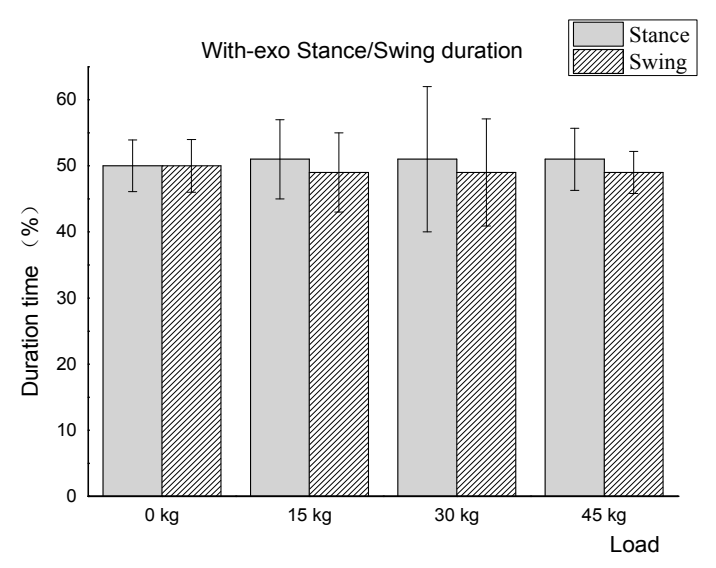

(b)

Figure 6. Comparison of gait phase stance and swing duration time. (a) without-Exo; (b) with-Exo.

\subsection{Unilateral Gait Report of with-Exo and without-Exo in a $45 \mathrm{~kg}$ Load Carriage Trial}

The unilateral gait report is a kind of time normalized gait cycle EMG pattern which can show the typical muscle activity characteristics and coordination of muscle groups during a walking trial. With appropriate test standardization, the averaged EMG pattern of gait cycles in a trial is highly reproducible. The averaged gait cycle data could be used to analyze the overall patterns and characteristics of the muscle activity within one gait cycle. It could be also used to compare the muscle activations under loaded (stance) and unloaded (swing) gait phases between with-Exo and without-Exo trials in the same load carriage condition.

The exoskeleton prototype was designed to augment human beings' performance of heavy load carriage. Therefore, a comprehensive case study was carried out on the heaviest load carriage condition, which was $45 \mathrm{~kg}$ load level, in order to access the loading performance of the exoskeleton prototype. The comparison was carried out between the conventional $45 \mathrm{~kg}$ carriage walking trials and exoskeleton-assisted $45 \mathrm{~kg}$ carriage walking trials.

The results are shown in Figure 7. Under the $45 \mathrm{~kg}$ carriage, the overall patterns and characteristics of the muscle activities were almost the same for the both with- or without-Exo load carriage conditions. In the loaded (stance) stage, the averaged activity of exoskeleton augmenting conditions were a little bit lower than the ones for conventional load walking conditions, except for the planter flexor PL. However, in the unloaded (swing) conditions, the curves' trends were almost contrary to the loaded (stance) phase. This situation complied with the poor adaptability and impedance effect from inertia. However, the load carriage performance was good. Considering the standard deviations, the conventional load carriage gait graphs are smoother than the assistive ones, as shown in Figure $7 \mathrm{~b}, \mathrm{c}$. 


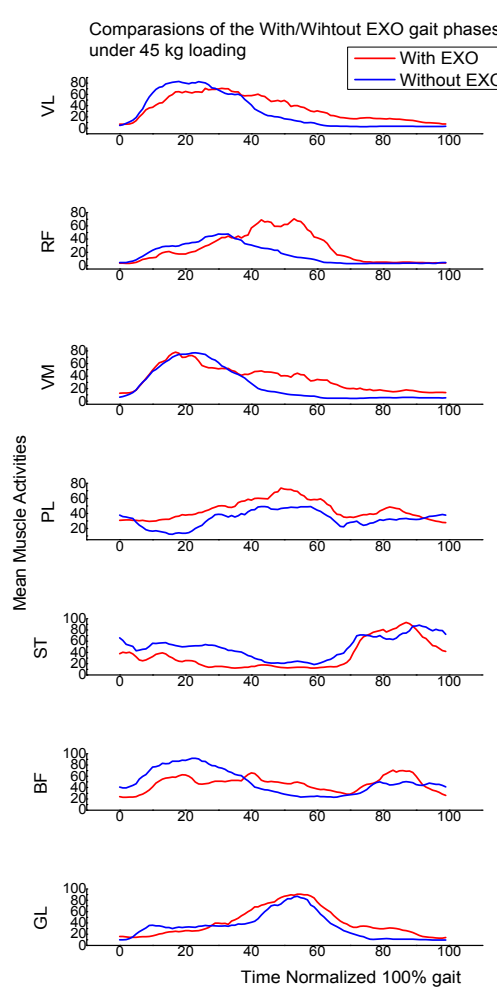

(a)
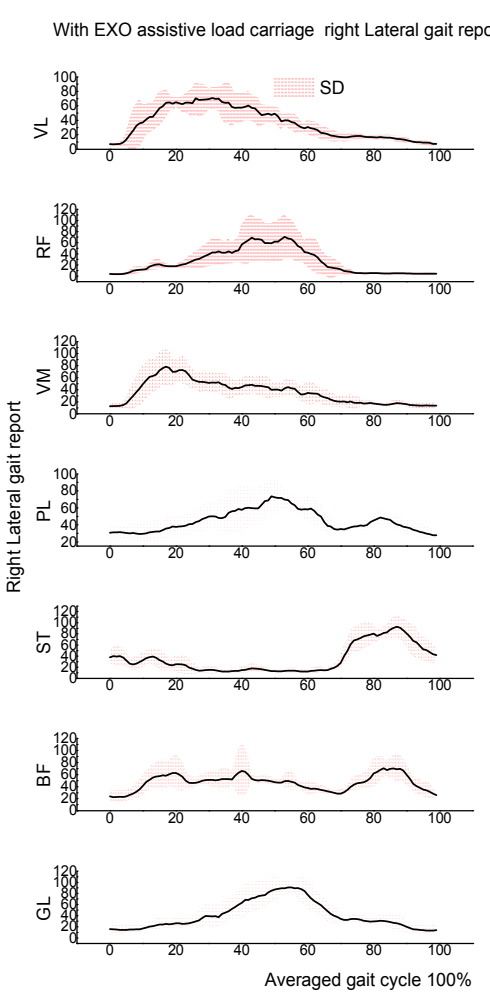

(b)
Conventional load carriage right Lateral gait report
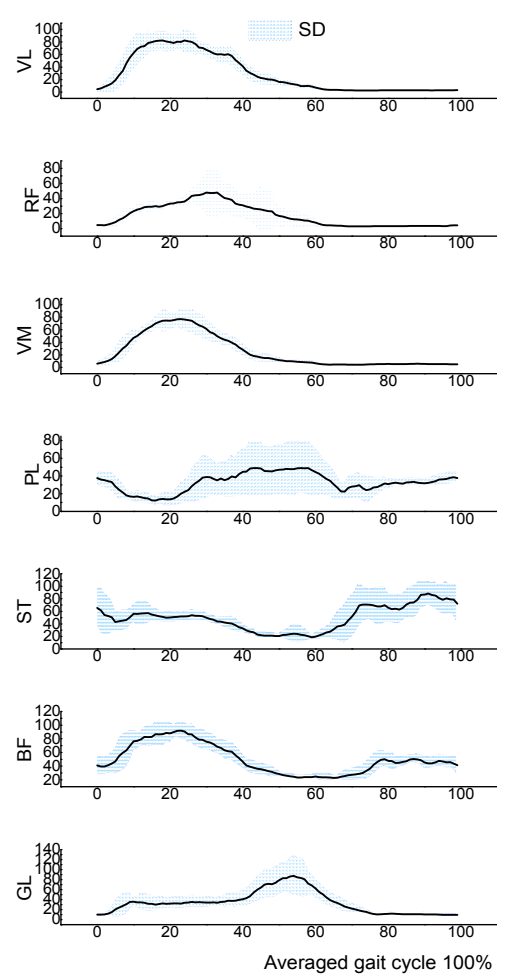

(c)

Figure 7. Time normalized EMG gait patterns for the measured right leg muscle groups during with-Exo and without-Exo walking in the case of $45 \mathrm{~kg}$ load carriage.

\subsection{Mean Muscle Activity}

As shown in Table 1, in the conventional carriage, the mean muscle activity of knee extensors $\mathrm{RF}, \mathrm{VM}$, and VL increased almost linearly with increasing of loads. The $\mathrm{R}^{2}$ were $0.917,0.992$, and 0.991 , respectively. However, there was no obvious change for knee flexor BF and ST. Additionally, the $\mathrm{R}^{2}$ were 0.829 and 0.562 , respectively. Relating to the load level, the changes of the planter flexor PL is linear.

Table 1. Mean Muscle Activities During $0 \mathrm{~kg}, 15 \mathrm{~kg}, 30 \mathrm{~kg}$, and $45 \mathrm{~kg}$ Load Levels Without the Exoskeleton. (Note: The determination coefficient $\mathrm{R}^{2}$ and the statistics $\mathrm{F}$ of Fischer used to confirm the linearity in whole cases are shown. The bold and italic style indicates linearity.)

\begin{tabular}{ccccccccc}
\hline \multirow{2}{*}{ Muscles } & \multicolumn{5}{c}{ Load (Mean Activities) } & \multirow{2}{*}{$\mathbf{F}$} & \multirow{2}{*}{$\mathbf{R}^{\mathbf{2}}$} & $\boldsymbol{p}$ \\
\cline { 2 - 5 } & $\mathbf{0 ~ \mathbf { ~ k g }}$ & $\mathbf{1 5} \mathbf{~ k g}$ & $\mathbf{3 0 ~} \mathbf{~ g}$ & $\mathbf{4 5} \mathbf{~ k g}$ & & & \\
\hline RF & 7.315 & 8.265 & 11.16 & 16 & $\mathbf{2 2 . 1 5}$ & $\mathbf{0 . 9 1 7}$ & $\mathbf{0 . 0 4 2}$ \\
VM & 10.02 & 13.95 & 18.5 & 23.9 & $\mathbf{3 9 3 . 0 0}$ & $\mathbf{0 . 9 9 2}$ & $\mathbf{0 . 0 0 3}$ \\
VL & 18.65 & 22.25 & 28.15 & 33.3 & $\mathbf{2 3 3 . 1 7}$ & $\mathbf{0 . 9 9 1}$ & $\mathbf{0 . 0 0 4}$ \\
BF & 4.45 & 4.35 & 4.94 & 5.215 & 9.71 & 0.829 & 0.09 \\
ST & 6.61 & 4.755 & 5.41 & 4.645 & 2.56 & 0.562 & 0.25 \\
PL & 7.675 & 8.935 & 10.28 & 14.4 & $\mathbf{1 9 . 2 4}$ & $\mathbf{0 . 9 0 6}$ & $\mathbf{0 . 0 4 8}$ \\
GL & 5.045 & 4.155 & 5.535 & 7.49 & 3.51 & 0.637 & 0.20 \\
\hline
\end{tabular}

However, in Table 2, the with-Exo conditions, RF, VM, and VL activities are not increasing with load level, as shown in Table 2. Some muscle's mean activities even decrease with heavier load level, such as the knee extensor muscles RF and VM. The PL muscle activities are far higher than the other muscles. 
Table 2. Mean Muscle Activities During 0 kg, 15 kg, 30 kg, and $45 \mathrm{~kg}$ Load Levels With the Exoskeleton. (Note: The determination coefficient $\mathrm{R}^{2}$ and the statistics $\mathrm{F}$ of Fischer used to confirm the linearity in whole cases are shown. The bold and italic style indicates linearity.).

\begin{tabular}{ccccccccc}
\hline \multirow{2}{*}{ Muscles } & \multicolumn{5}{c}{ Load (Mean Activities) } & \multirow{2}{*}{ F } & \multirow{2}{*}{$\mathbf{R}^{\mathbf{2}}$} & $\boldsymbol{p}$ \\
\cline { 2 - 5 } & EXO + 0 & EXO + 15 & EXO + 30 & EXO + 45 & & & \\
\hline RF & 16.35 & 23.15 & 25.8 & 24.35 & 4.17 & 0.68 & 0.17 \\
VM & 19.2 & 18.15 & 21.45 & 26.2 & 6.68 & 0.77 & 0.12 \\
VL & 26.1 & 20.45 & 22.4 & 22.2 & 0.78 & 0.28 & 0.47 \\
BF & 4.81 & 6.28 & 6.57 & 8.285 & $\mathbf{3 2 . 4 0}$ & $\mathbf{0 . 9 4}$ & $\mathbf{0 . 0 3}$ \\
ST & 4.39 & 4.94 & 7.96 & 8.81 & $\mathbf{2 4 . 1 2}$ & $\mathbf{0 . 9 2}$ & $\mathbf{0 . 0 4}$ \\
PL & 22.8 & 31.5 & 53.75 & 65.5 & $\mathbf{7 2 . 3 4}$ & $\mathbf{0 . 9 7}$ & $\mathbf{0 . 0 1 4}$ \\
GL & 10.75 & 11.915 & 10.81 & 12.76 & 1.54 & 0.43 & 0.34 \\
\hline
\end{tabular}

Most of the muscles' mean activities increased when using the assistive exoskeletons, especially in the planter flexor PL $(p<0.001)$, as shown in Figure 8. As far as knee flexors BF and ST were concerned, although the mean activities are higher than the conventional condition, there were no obvious change to the muscle activities with increasing loads. According to the experiment, it could be concluded that the flex process was seriously affected by the exoskeleton. For the plantar flexor muscles PL and GL, much more activity was needed when using the EXO, especially for PL. It may be caused by the exoskeleton self-weight and adaptability.

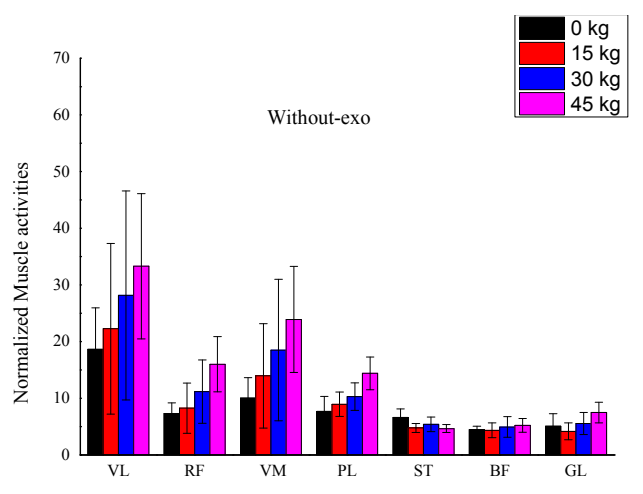

(a)

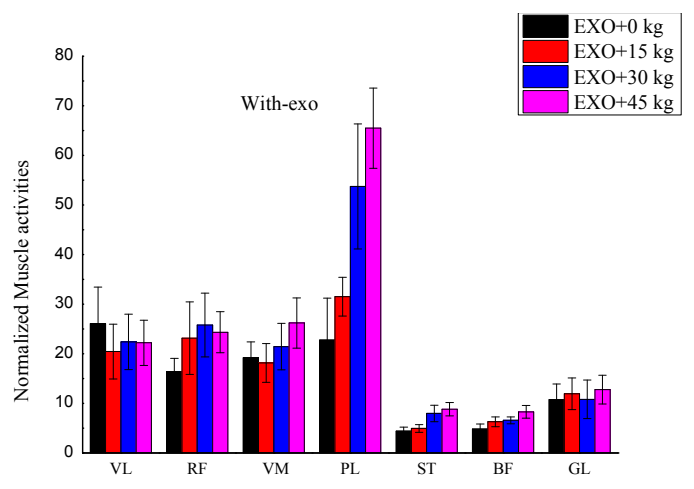

(b)

Figure 8. Normalized averaged muscle activities of with-Exo and without-Exo load carriage walking.

\subsection{Main and Two-Way ANOVA Interaction Effects of with or without * Load Level of EMG Values}

A summary of ANOVA for the effects of load (L) with- or without-Exo (W) for nRMS values of nEMG data are given in Table 3. The two-way ANOVA method was used in the calculations. Significant changes of the load main effects in nRMS are found for almost all the measured muscles $(p<0.05)$, except for the muscle BF $(p>0.05)$. nRMS values greatly increased over load levels in the knee extensors RF, VM, and VL $(p<0.001)$. The main effects of with- or without-EXO are significantly different for almost all the muscles $(p<0.05)$, except for the muscle VM $(p>0.05)$. Load ${ }^{*}$ with or without interaction effects were significant in some muscles such as RF, PL, and GL $(p<0.05)$.

The main and interactive effects could also be seen from plots of Figure 9. Although the overall characteristic is that the mean nRMS from the exoskeleton assisted load walking was higher than those from the conventional load walking, almost all the rates of nRMS that increased during the exoskeleton assisted load walking slowed down with the increasing of load level. Even in some muscles the rates are decreasing with the increase of the load level, such as muscles BF, ST, and GL. Especially for the loaded muscle VM, when the load level is over $30 \mathrm{~kg}$, the exoskeleton augmentation potential appears. 
Table 3. Summary of Two-Way ANOVA Results for the Main and Interaction Effects of With or Without and Load Final EMG Values. $\mathrm{W} \times \mathrm{L}$ stands for with or without and load interaction. F means $\mathrm{F}$ test value. Lower-case $p$ means statistical significance and the significant differences were bolded.

\begin{tabular}{|c|c|c|c|}
\hline Muscles & $\begin{array}{l}\text { With- or Without-EXO } \\
\text { (Main Effects) } \\
\text { F (p) }\end{array}$ & $\begin{array}{c}\text { Load Level } \\
\text { (Main Effects) } \\
\text { F ( } p)\end{array}$ & $\begin{array}{c}\mathrm{W} \times \mathrm{L} \\
\text { (Interaction Effects) } \\
\mathrm{F}(p)\end{array}$ \\
\hline RF & $6.418(p=0.017)$ & $26.124(p=0.000)$ & $3.356(p=0.04)$ \\
\hline VM & $0.058(p=0.810)$ & $16.320(p=0.000)$ & $0.734(p=0.427)$ \\
\hline VL & $6.373(p=0.017)$ & $19.836(p=0.000)$ & $1.185(p=0.312)$ \\
\hline $\mathrm{BF}$ & $22.653(p=0.000)$ & $1.407(p=0.250)$ & $2.472(p=0.078)$ \\
\hline ST & $12.637(p=0.001)$ & $5.240(p=0.005)$ & $2.767(p=0.062)$ \\
\hline PL & $15.496(p=0.000)$ & $6.728(p=0.005)$ & $4.158(p=0.031)$ \\
\hline GL & $21.391(p=0.000)$ & $9.287(p=0.000)$ & $6.729(p=0.003)$ \\
\hline
\end{tabular}
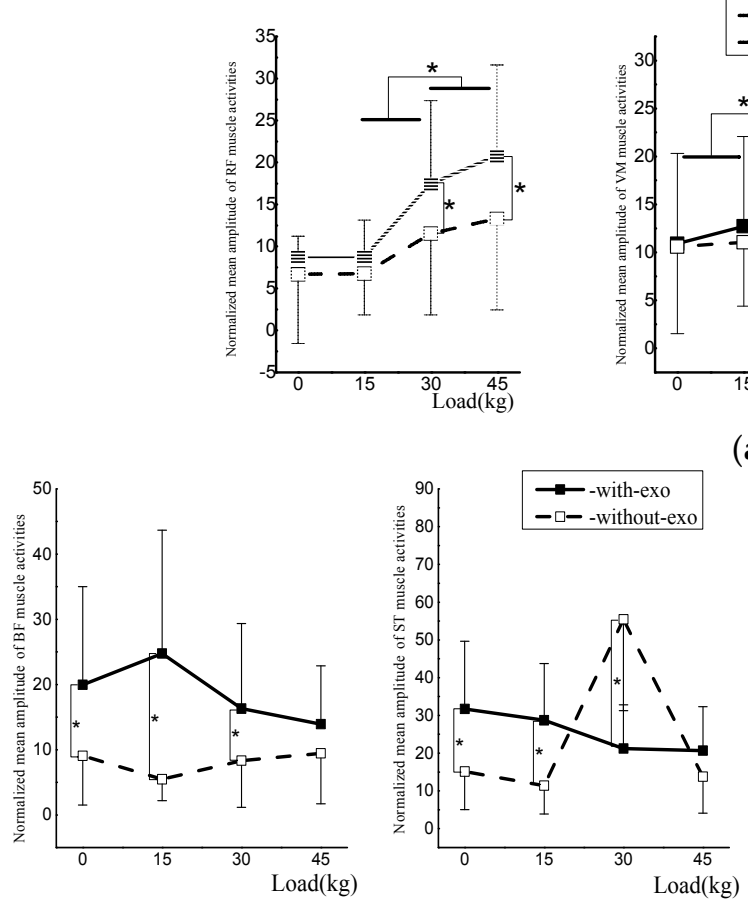

(b)

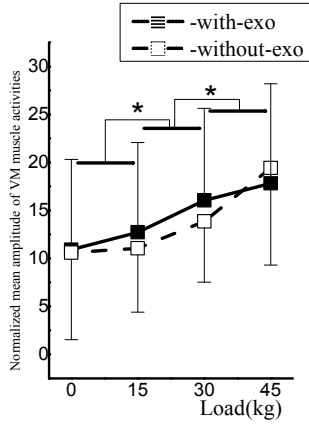

(a)
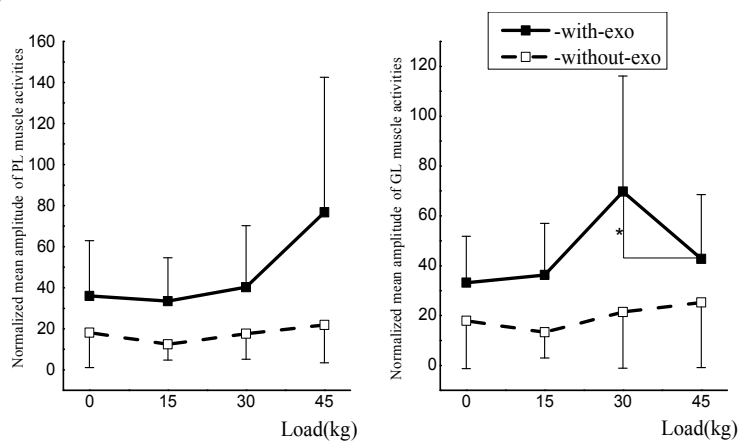

(c)

Figure 9. With-Exo or without-Exo and four loading levels effects on initial values of normalized RMS (nRMS) for (a) the right knee extensors (RF, VM, VL); (b) for the right knee flexors (BF, ST); (c) the right plantar flexors (PL, GL). The star $\left({ }^{*}\right)$ indicates the significant difference $(p<0.001)$ between two related factors.

\subsection{The Discomfort Index and Six Indices of NASA-TXL Rating Scales}

For the subjective performance, the two primary independent factors were still: (1) without-EXO and with-EXO carrying condition and (2) the four loading levels. The dependent experiment variables were: (1) the discomfort rating $C$ and (2) the group weighted ratings of the NASA TLX scales (MD, PD, TD, OP, EF, FR). Because there were just nine investigated samples, the data abnormality was tested by boxplot. The Shapiro-Wilk method was used to test the data normality. A Levene variance homogeneity test was used. The results indicated that there were no abnormal data. The residual errors were normal $(p>0.05)$.

According to the literature [19], this experiment is a kind of Continuous SINGLE-axis MANUAL task. Considering the fact that the TLX index was developed for significantly more complex tasks, the 
combined numeric value of the TLX is too coarse to be sensitive to the relatively small variations in the task elements. Therefore, the effect of "with or without" variation on the individual mean discomfort rating scales is shown in Figure 10a. Additionally, the individual mean MD, PD, TD, OP, EF, and FR rating scales are shown in Figure 10b. It is noticed that in the conventional load carriage, the workload demands for all effort required and the extended discomfort $C$ for $0 \mathrm{~kg}$ level were defined as " 1 ". With increasing load level, the subjective discomfort increased linearly and significantly in the conventional carriage, while in the exoskeleton assisted carriage condition, the discomfort increased much slower. Similar trends for the standard six rating scales could also be observed in Figure 10b.

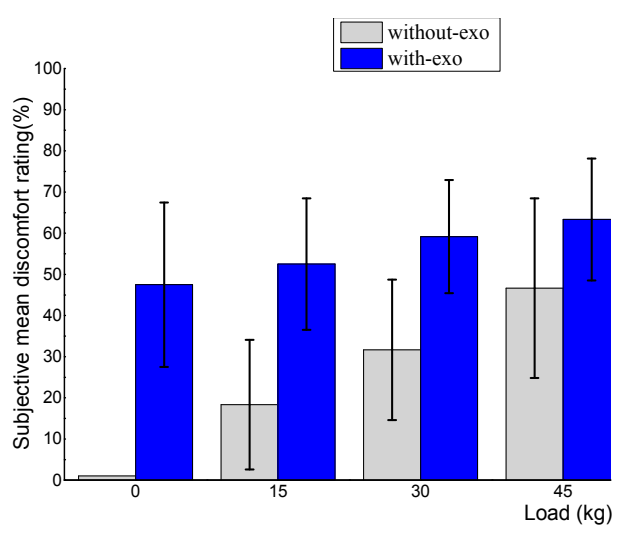

(a)

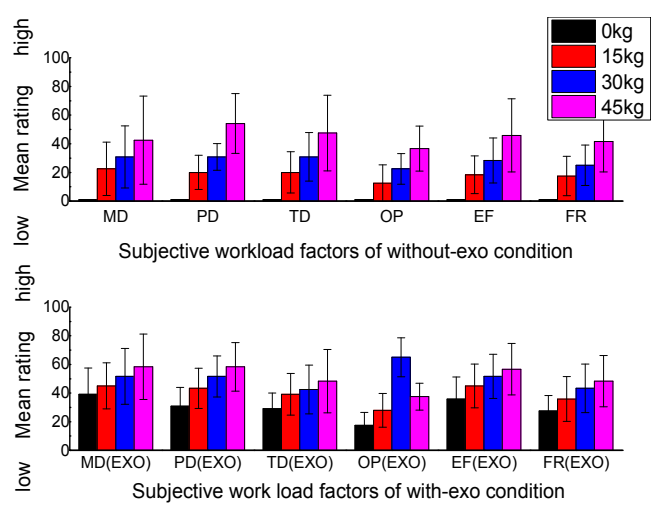

(b)

Figure 10. (a) The subjective discomfort performance. (b) The comparisons of mean rating value plots of the six workload factors.

\subsection{The Weighting Factor and the Results}

The mean group weighting factors of the two carriage conditions were determined for the NASA TLX rating scales after the experiment, as shown in Figure 11. The results of the paired $t$-test illustrate that the MD and PD weights are significantly different in the two carriage conditions. The MD weight in the with-EXO condition is significantly higher $(p=0.037)$ than the one which is in the conventional carriage. It means the assistive exoskeleton increases the mental demand of the subjects. According to the experiment process, the reason may be the subjects have to stabilize the human-robot system not only by providing force and torque, but by providing mental control strategies. As far as PD was concerned, the PD weight in the with-EXO condition is significantly lower $(p=0.005)$ than the one in the conventional carriage. It means the assistive exoskeleton successfully shared the loadings in the experiment process. There were no significant differences for the weighting factors TD, OP, and EF. For the weighting factor FR, it indicated that subjects were more insecure, discouraged, irritated, stressed, and annoyed with exoskeleton assisted loading walking. This indicates a poor ergonomic user interface for the exoskeleton.

According to the comparison of weight factors, the discomfort $C$, mental demand MD, physical demand PD, and frustration FR were selected into the two-way ANOVA analysis. The results are shown in Table 4. Although there is no interaction effect in the statistical results of Discomfort, Mental Demand, and Physical Demand with carrying condition and load level, the ordinal interactions existed in the interaction plot, as shown in Figure 12. In reality, soldiers often carry loads over $55 \mathrm{~kg}$. If the load level was increased, a disordinal interaction may have appeared. The with- or without-EXO main effects on the three workload factors were significantly different $(p<0.0001)$. The load level main effects on the Physical Demand were significantly different $(p<0.0001)$, and also on Discomfort and Mental Demand. The "with or without" main effects were pair-wise compared. The unweight marginal mean values were $31.208 \pm 5.502, p<0.0001$. The with-Exo discomfort level was higher $21.438(95 \% \mathrm{CI}$ is from $20.890-42.328)$ than the without-Exo. 


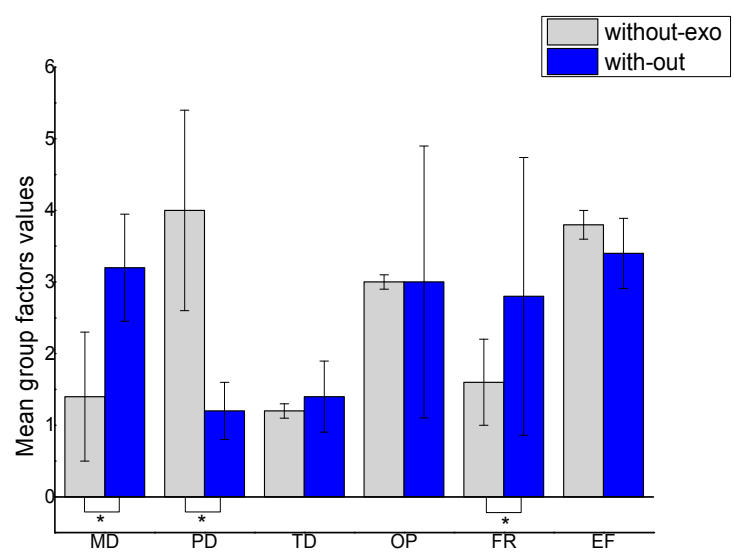

Figure 11. The comparison of weight factors between without-exo and with-exo walking conditions.

Table 4. Summary of Two-Way ANOVA Results for the Main and Interaction Effects on the Discomfort, Mental Demand and Physical Demand. (Note: The significant differences were indicated as bold and italic style. F means F test value. Lower-case $p$ indicates statistical significance.)

\begin{tabular}{cccc}
\hline $\begin{array}{c}\text { NASA-TLX Rating } \\
\text { Scale Values }\end{array}$ & $\begin{array}{c}\text { With- or Without-EXO } \\
\text { (Main Effects) } \\
\text { F }(\boldsymbol{p})\end{array}$ & $\begin{array}{c}\text { Load Levels (L) } \\
\text { (Main Effects) } \\
\mathbf{F}(\boldsymbol{p})\end{array}$ & $\begin{array}{c}\text { W } \times \text { L } \\
\text { (Interaction Effects) } \\
\mathbf{F}(\boldsymbol{p})\end{array}$ \\
\hline Discomfort & $\mathbf{3 2 . 1 7 7}(<\mathbf{0 . 0 0 0 1 )}$ & $\mathbf{5 . 7 6 4 ( < 0 . 0 5 )}$ & $1.288(=0.292)$ \\
Mental Demand & $\mathbf{1 4 . 5 1 3 ( < 0 . 0 0 0 1 )}$ & $\mathbf{4 . 0 3 0}(<\mathbf{0 . 0 5 )}$ & $0.570(=0.638)$ \\
Physical Demand & $\mathbf{2 0 . 0 0 6 ( < 0 . 0 0 0 1 )}$ & $\mathbf{1 5 . 0 0 8}(<\mathbf{0 . 0 0 0 1 )}$ & $1.564(=0.213)$ \\
\hline
\end{tabular}

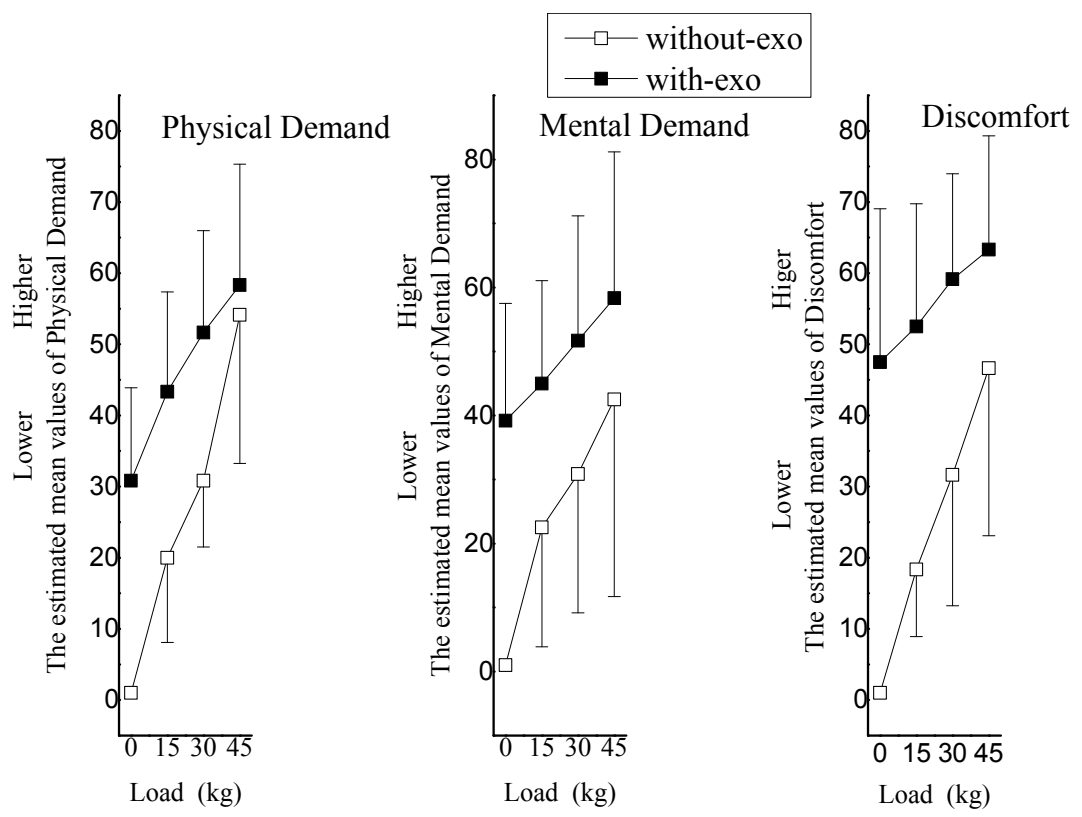

Figure 12. The interaction trend plot of with-Exo or without-Exo $\times$ load level.

\section{Discussion}

Regular load carriage often happens in specific situations, such as for soldiers, school children, hikers, and assembly line workers. It was one of the most frequent risk factors for MSDs which have been confirmed by EU-OSHA. Although a few exoskeletons have been developed to decrease the loading effects on the human body, there is still lacking evidence that these assistive devices allow human beings to move or walk freely or make carriage-loaded MSDs decrease. In this study, one exoskeleton prototype was examined as a potential ergonomic device for application 
in regular loading tasks. Some advices should be provided to improve the ergonomic performance and human-robot interaction performance so as to decrease carriage-loaded MSDs.

A few assistive exoskeletons for the lower extremities are already commercially available. Some have been introduced into rehabilitation centers, industry assembly lines, and military training [20-22]. Some measures and analyses on the metabolic effects, muscle activities, and human gait patterns have been carried out on the related exoskeletons $[11,23,24]$. This study remains one of the first to quantify muscle activities for which the exoskeletons are not specifically designed to decrease the metabolic cost. In particular, this investigation aims to quantify muscle amplitude and activities that result from performing four levels of load carriage walking on a treadmill, with and without an exoskeleton. At the same time, this study examined the subjective performance of the exoskeleton using the extended NASA-TLX questionnaire.

Most research has examined muscle activities of the lower limb, comparing the mean and peak amplitude from free treadmill walking to exoskeleton treadmill walking, and have reported a significant increase in muscle activity with the exoskeleton [24]. Few studies have reported decreased muscle activity when using an exoskeleton [25]. It is important to note that the existing augmenting exoskeletons have reported no decrease in muscle activity and oxygen consumption [11,20]. However, this is not to say the assistive exoskeletons pose no use to human beings. It is reported that any real metabolic advantage imparted by the EXO may be dependent on the magnitude of the load carried, such that the metabolic energy cost per unit of mass becomes lower than non-EXO assisted load carriage only when heavy loads (greater than $50 \%$ of body mass) are carried by the device [11]. The results of this study are consistence with the former mentioned results. As shown in Figure 9, the interaction of the Load level $\times$ with or without-EXO should happen with increasing of load level.

When comparing the load level main effects on muscle activity, it is illustrated that the structure of the effects are different for the with- and without-Exo walking conditions. By looking further into the research results, we can see in the loaded (stance) gait stage, muscle activities decrease with same load level for the with-Exo walking condition. However, in the unloaded (swing) gait stage, the muscle activities increase. This result suggests that the assistive exoskeleton could effectively share the load with human beings. However, exoskeleton weight and adaptability are not yet good enough. The structure design and control strategy should be reconsidered from an ergonomic point of view to improve the agility, ease of push-off, and adaptability of human-robot systems, or the assistive devices could form a new risk factor for MSDs from loaded carriage.

Loading of the lower limb is increased when carrying load. Therefore, a longer ground contact time would increase the amount of time the limb is subjected to increased loading. This may result in increased loading of lower limb structures, such as increased metatarsal compression [24]. Alternatively, a longer ground contact time may be a protective mechanism to lower the rate of loading on the internal structures in order to reduce the potential negative effects of increased load magnitude. It is therefore unclear whether a longer ground contact time is associated with the development of injury, or if it is a mechanism to minimize the risk of injury. Therefore, interventions for increasing muscular strength and endurance of the plantar flexor and knee extensor muscles may be beneficial [2].

EMG data indicated increased ankle plantar, flexor, and knee extensor muscle activity whilst carrying load, in support of the hypotheses. The use of the exoskeleton most significantly increased max and mean muscle activities in the ankle plantar flexor PL in loaded carriage. A significant increase in muscle activation also happened for the knee extensor and hip flexor RF, and the knee flexor and hip extensor ST. However, these significant differences could not be found for the other four pair-wise comparisons (VL, VM, BF, and GL).

The very high rates of PL (peroneus longus) muscle activity which were observed may have been the result of a greater contribution of the plantar flexor muscles while knee extensor moments were reduced. Plantar flexor muscle activation has previously been associated with both tibia and metatarsal stress fractures [26]. The suggested interventions to reduce fatigability of the plantar flexor muscles during load carriage activity should be considered, since there is no activation in the exoskeleton ankle 
joint. Furthermore, the observed significant increases in PL muscle activity which appeared in the exoskeleton assisted load carriage is more likely a result of the loose fastening, rigidity, and inertia of the exoskeleton shoes. Therefore, the structure of exoskeleton shoes and the human-robot shoe interface should be redesigned.

In the early stance, the quadriceps muscles act to control knee flexion, while the plantar flexors act at the end of stance to contribute to push-off [27]. In theory, carrying heavier loads is more demanding on the knee joint musculature than that of the ankle. Therefore, the exoskeleton prototype is designed to be activated only in the knee joints. However, the increased GL and PL muscle activity suggests a greater plantar flexor force production is required for push-off when carrying a load of $45 \mathrm{~kg}$, as shown in Figure 4. The activation of the ankle joints is significant.

It is clear from the results that load level has a strong influence on the subject's discomfort. The exoskeleton itself also seriously affects the subject's discomfort. However, during the trials with assistance of the exoskeleton, the subject's discomfort increased more slowly with increasing load level. When asked directly, the participants preferred to use the exoskeleton at the heavier load levels of $30 \mathrm{~kg}$ and $45 \mathrm{~kg}$. Although no more than a $45 \mathrm{~kg}$ load level was tested, ordinal interactions existed in the interaction plot, as shown in Figure 10. That means if the load level was added, the disordinal interaction may appear.

Considering the six standard NASA-TLX indices, the load level also has stronger influence on the subject's performance metrics than the with- or without-Exo has. However, during the with-EXO load carriage, the MD weight is higher and PD weight is lower than during the without-EXO load carriage. This means the exoskeleton increased the mental demand and decreased the physical demand. This situation is consistent with the experiment experience. The subjects reported they had to pay attention to the push-off of the swing for the exoskeleton and the stabilization of the overall system. Furthermore, there existed an interaction force about the lower limb which the subjects had to counteract. This again verified that the adaptability and human-robot interaction performance have to be improved, or the extra risk factors for MSDs may arise for the exoskeleton wearers.

In order to actually help the human being in heavy load carriage and decrease muscle activities, a better ergonomic mechanical structure should be proposed for the tested prototype. Especially for the ankle or foot parts, power should be supplied and the human-exoskeleton connections should be reconsidered. At that point, human beings should be included in the control scheme of the human-exoskeleton system.

\section{Conclusions}

Although the activities of the knee extensors VM and VL decreased with the help of the powered exoskeleton under the heaviest carriage level, this study indicated much stronger muscular activities of most tested muscles when carrying loads using the assistive exoskeleton, especially the planter flexor PL. These increased muscular responses may result in new muscular skeletal injuries. The subjective comfort performance was lower than that in the conventional carriage condition. The findings show that the argumentation exoskeleton can help reduce the burden of the load, while stabilization and recruitment are severely mentally and physically demanding. This is consistent with the investigation results of more mental demands and lower physical demands in the NASA-TLX factors. Such investigations may have important implications related to the construction design and human-robot control strategies of an augmentation exoskeleton. Some of the results in this article may possibly be used as empirical data for sensorimotor interactions when walking with different types of exoskeletons.

Author Contributions: H.L. and W.C. conceived and designed the experiments. H.L. performed the experiments, analyzed the data, and wrote the paper. F.L. provided suggestions for the experiments. M.Z. contributed the analysis tools. K.W. provided management of the experiments.

Funding: This work was supported by Chinese National Science Foundation Grant Number 51675450. We are especially grateful to the participant for the volunteers.

Conflicts of Interest: The authors declare no conflict of interest. 


\section{References}

1. Hamzat, T.K.; Abdulkareem, T.A.; Akinyinka, O.O.; Fatoye, F.A. Backpack-related musculoskeletal symptoms among nigerian secondary school students. Rheumatol. Int. 2014, 34, 1267-1273. [CrossRef] [PubMed]

2. Rice, H.; Fallowfield, J.; Allsopp, A.; Dixon, S. Influence of a 12.8-km military load carriage activity on lower limb gait mechanics and muscle activity. Ergonomics 2017, 60, 649-656. [CrossRef] [PubMed]

3. Anderson, A.M.; Meador, K.A.; Mcclure, L.R.; Makrozahopoulos, D.; Brooks, D.J.; Mirka, G.A. A biomechanical analysis of anterior load carriage. Ergonomics 2007, 50, 2104-2117. [CrossRef] [PubMed]

4. Knapik, J.; Reynolds, K.; Staab, J.; Vogel, J.A.; Jones, B. Injuries associated with strenuous road marching. Mil. Med. 1992, 157, 64-67. [CrossRef] [PubMed]

5. Lavender, S.A.; Ko, P.L.; Sommerich, C.M. Biomechanical evaluation of the eco-pick lift assist: A device designed to facilitate product selection tasks in distribution centers. Appl. Ergon. 2013, 44, 230-236. [CrossRef]

6. Kazerooni, H. The berkeley lower extremity exoskeleton. J. Dyn. Syst. Meas. Control 2006, 128, 9-15. [CrossRef]

7. Kawamoto, H.; Taal, S.; Niniss, H.; Hayashi, T.; Kamibayashi, K.; Eguchi, K. Voluntary motion support control of robot suit HAL triggered by bioelectrical signal for hemiplegia. In Proceedings of the International Conference of the IEEE Engineering in Medicine and Biology, Buenos Aires, Argentina, 31 August-4 September 2010; pp. 462-466.

8. Panizzolo, F.A.; Ignacio, G.; Asbeck, A.T.; Christopher, S.; Kai, S.; Holt, K.G. A biologically-inspired multi-joint soft exosuit that can reduce the energy cost of loaded walking. J. Neuroeng. Rehabil. 2016, 13, 43. [CrossRef]

9. Dollar, A.M.; Herr, H. Lower extremity exoskeletons and active orthoses: Challenges and state-of-the-art. IEEE Trans. Robot. 2008, 24, 144-158. [CrossRef]

10. Schiffman, J.M.; Gregorczyk, K.N.; Bensel, C.K.; Hasselquist, L.; Obusek, J.P. The effects of a lower body exoskeleton load carriage assistive device on limits of stability and postural sway. Ergonomics 2008, 51, 1515-1529. [CrossRef]

11. Gregorczyk, K.N.; Obusek, J.P.; Hasselquist, L.; Bensel, J.S.M.; Carolyn, K.; Gutekunst, D. The Effects of a Lower Body Exoskeleton Load Carriage Assistive Device on Oxygen Consumption and Kinematics during Walking with Loads; U.S. Army Natick Soldier Center: Natick, MA, USA, 2006.

12. Zanotto, D.; Akiyama, Y.; Stegall, P.; Agrawal, S.K. Knee joint misalignment in exoskeletons for the lower extremities: Effects on user's gait. IEEE Trans. Robot. 2015, 31, 978-987. [CrossRef]

13. Aguirre-Ollinger, G.; Colgate, J.E.; Peshkin, M.A.; Goswami, A. Inertia compensation control of a onedegree-of-freedom exoskeleton for lower-limb assistance: Initial experiments. IEEE Trans. Neural Syst. Rehabil. Eng. 2012, 20, 68-77. [CrossRef]

14. Ye, D.; Galiana, I.; Asbeck, A.; Rossi, S.D.; Bae, J.; Santos, T. Biomechanical and physiological evaluation of multi-joint assistance with soft exosuits. IEEE Trans. Neural Syst. Rehabil. Eng. 2017, 25, 119-130.

15. Hansen, C.; Gosselin, F.; Ben, K.M.; Devos, P.; Marin, F. Design-validation of a hand exoskeleton using musculoskeletal modeling. Appl. Ergon. 2018, 68, 283-288. [CrossRef] [PubMed]

16. Michalos, G.; Karvouniari, A.; Dimitropoulos, N.; Togias, T.; Makris, S. Workplace analysis and design using virtual reality techniques. Cirp Ann.-Manuf. Technol. 2018, 67, 141-144. [CrossRef]

17. Liu, F.; Cheng, W.; Wu, Q. Research on human exoskeleton based on rigid-flexible coupling system. Mech. Sci. Technol. Aerosp. Eng. 2013, 32, 15.

18. Zhang, M.K.; Cheng, W.; Li, H.X. Drive of the powered exoskeleton and driving compensation with the human-machine coupling interaction while squatting. Robot 2017, 39, 514-522.

19. Hart, S.G.; Staveland, L.E. Development of nasa-tlx (task load index): Results of empirical and theoretical research. Adv. Psychol. 1988, 52, 139-183.

20. Hidler, J.W.; Wisman, N. Kinematic trajectories while walking within the Lokomat robotic gait-orthosis. Clin. Biomech. 2008, 23, 1251-1259. [CrossRef]

21. Stegall, P.; Winfree, K.; Zanotto, D.; Agrawal, S.K. Rehabilitation exoskeleton design: Exploring the effect of the anterior lunge degree of freedom. IEEE Trans. Robot. 2013, 29, 838-846. [CrossRef]

22. Suzuki, K.; Mito, G.; Kawamoto, H.; Hasegawa, Y.; Sankai, Y. Intention-based walking support for paraplegia patients with robot suit hal. Adv. Robot. 2007, 21, 1441-1469. 
23. Knaepen, K.; Beyl, P.; Duerinck, S.; Hagman, F.; Lefeber, D.; Meeusen, R. Human-robot interaction: Kinematics and muscle activity inside a powered compliant knee exoskeleton. IEEE Trans. Neural Syst. Rehabil. Eng. 2014, 22, 1128-1137. [CrossRef] [PubMed]

24. Del-Ama, A.J.; Asín-Prieto, G.; Piñuela-Martín, E.; Pérez-Nombela, S.; Lozano-Berrio, V.; Serrano-Muñóz, D. Muscle Activity and Coordination During Robot-Assisted Walking with H2 Exoskeleton. In Converging Clinical and Engineering Research on Neurorehabilitation II, 1st ed.; Jaime, I., José, G.V., José, M.A., Metin, A., José, L.P., Eds.; Springer International Publishing: Segovia, Spain, 2017; Volume 15, pp. 349-353, ISBN 978-3-319-46668-2.

25. Collins, S.H.; Wiggin, M.B.; Sawicki, G.S. Reducing the energy cost of human walking using an unpowered exoskeleton. Nature 2015, 522, 212-215. [CrossRef] [PubMed]

26. Arndt, A.; Ekenman, I.; Westblad, P.; Lundberg, A. Effects of fatigue and load variation on metatarsal deformation measured in vivo during barefoot walking. J. Biomech. 2002, 35, 621-628. [CrossRef]

27. Nindl, B.C.; Harman, E.A.; Marx, J.O.; Gotshalk, L.A.; Frykman, P.N.; Lammi, E. Regional body composition changes in women after 6 months of periodized physical training. J. Appl. Physiol. 2000, 88, 2251-2259. [CrossRef] [PubMed]

(C) 2018 by the authors. Licensee MDPI, Basel, Switzerland. This article is an open access article distributed under the terms and conditions of the Creative Commons Attribution (CC BY) license (http://creativecommons.org/licenses/by/4.0/). 